\title{
Characterization of CTX-M ESBLs in Enterobacter cloacae, Escherichia coli and Klebsiella pneumoniae clinical isolates from Cairo, Egypt
} Noha G Khalaf*1, Mona M Eletreby ${ }^{2}$ and Nancy D Hanson ${ }^{3}$

Address: ${ }^{1}$ Department of Microbiology and Immunology, Faculty of Pharmacy Helwan University, Helwan, Egypt, ${ }^{2}$ Clinical Microbiology department, National Heart Institute, Cairo, Egypt and ${ }^{3}$ Center for Research in Anti-Infectives and Biotechnology, Department of Medical Microbiology and Immunology, Creighton University School of Medicine, Omaha, Nebraska, USA

Email: Noha G Khalaf* -ng_khalaf@yahoo.com; Mona M Eletreby - meltreby@hotmail.com; Nancy D Hanson - nancyhanson@creighton.edu

* Corresponding author

Published: 4 June 2009

BMC Infectious Diseases 2009, 9:84 doi:10.1 186/1471-2334-9-84
Received: 4 August 2008

Accepted: 4 June 2009

This article is available from: http://www.biomedcentral.com/I47/-2334/9/84

(C) 2009 Khalaf et al; licensee BioMed Central Ltd.

This is an Open Access article distributed under the terms of the Creative Commons Attribution License (http://creativecommons.org/licenses/by/2.0), which permits unrestricted use, distribution, and reproduction in any medium, provided the original work is properly cited.

\begin{abstract}
Background: A high rate of resistance to $3^{\text {rd }}$ generation cephalosporins among Enterobacteriaceae isolates from Egypt has been previously reported. This study aims to characterize the resistance mechanism (s) to extended spectrum cephalosporins among resistant clinical isolates at a medical institute in Cairo, Egypt.

Methods: Nonconsecutive Klebsiella pneumoniae (Kp), Enterobacter cloacae (ENT) and Escherichia coli (EC) isolates were obtained from the clinical laboratory at the medical institute. Antibiotic susceptibility was tested by CLSI disk diffusion and ESBL confirmatory tests. MICs were determined using broth microdilution. Isoelectric focusing (IEF) was used to determine the pl values, inhibitor profiles, and cefotaxime (CTX) hydrolysis by the $\beta$-lactamases. PCR and sequencing were performed using bla $a_{\mathrm{CTX}-\mathrm{M}}$ and ISEcp I-specific primers, with DNA obtained from the clinical isolates. Conjugation experiments were done to determine the mobility of bla $a_{\text {CTX-M. }}$.

Results: All five clinical isolates were resistant to CTX, and were positive for ESBL screening. IEF revealed multiple $\beta$-lactamases produced by each isolate, including a $\beta$-lactamase with a pl of 8.0 in $\mathrm{Kp}$ and ENT and a $\beta$-lactamase with a pl of 9.0 in EC. Both $\beta$-lactamases were inhibited by clavulanic acid and hydrolyzed CTX. PCR and sequence analysis identified bla $a_{\text {CTX-M-14 }}$ in $K_{P}$ and ENT and a bla $_{\mathrm{CTX}-\mathrm{M}-15}$ in EC. Both bla $a_{\mathrm{CTX}-\mathrm{M}-14}$ and bla $a_{\mathrm{CTX}-\mathrm{M}-15}$ were preceded by ISECP/ elements as revealed by partial sequence analysis of the upstream region of the bla ${ }_{\mathrm{CTX}-\mathrm{M}}$ genes. bla $a_{\mathrm{CTX}-\mathrm{M}-15}$ was transferable

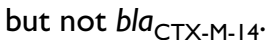

Conclusion: This is the first report of CTX-M-I4 in Kp and ENT isolates from Egypt, the Middle East and North Africa.

\section{Background}

Recent studies on Enterobacteriaceae isolates from Egypt have reported a resistance rate to third generation cepha- losporins of $70 \%[1,2]$. A survey, carried out in 20012002 and covered medical centers in Northern and Southern European countries, Egypt, Lebanon, Saudi Arabia 
and South Africa, reported the highest incidence of extended spectrum $\beta$-lactamases (ESBLs)-producing isolates in Egypt [3].

CTX-M ESBLs are the most prevalent ESBLs worldwide [4]. Recently, CTX-M ESBLs have been reported in Egypt [5], with CTX-M-15 being the most common ESBL reported in the Middle East region and North Africa $[6,5,7]$. However, CTX-M-14 has also been detected in Escherichia coli isolates from Egypt and Tunisia [5,8]. But CTX-M-14 has not been reported in Klebsiella pneumoniae isolates in this geographical region before.

CTX-Ms are class A ESBLs that are most active against cefotaxime [9]. However, some CTX-Ms can hydrolyze ceftazidime such as CTX-M-15 and CTX-M-19 [10,11]. The nucleotide sequences of $b l a_{\text {СтX-м }}$ genes are highly related to the nucleotide sequence of Kluyvera spp. $[12,13]$.

Clinical isolates of K. pneumoniae, Enterobacter cloacae and E. coli were sent from the clinical microbiology laboratory in a medical institute in Cairo, Egypt to investigate the mechanism (s) responsible for resistance to extended spectrum cephalosporins.

\section{Methods}

\section{Bacterial strains}

Five clinical isolates were sent on blood agar plates from the clinical laboratory at the medical institute. The isolates were three nonconsecutive $K$. pneumoniae isolates and one E. coli isolate, which were collected from chest wound swabs from patients in an adult surgical ICU ward. In addition, one E. cloacae isolate was obtained from central venous line of a patient in the pediatric ICU ward. Informed written consents were obtained from patients. Identification of the isolates was performed using Phoenix $^{\circledast}$ bacterial identification panels (NMIC/ID-107) and $\mathrm{API}^{\oplus} 20 \mathrm{E}$ strips (Biomerieux SA, Marcy-l'Etoile, France).

\section{Susceptibility test}

Antibiotic susceptibility was tested using disk diffusion with the following drugs: cefotaxime, ceftazidime, tetracycline, gentamicin, amikacin, ciprofloxacin, and sulfamethoxazole. ESBL production was investigated using cefotaxime and ceftazidime, alone and in combination with clavulanic acid (BBL, Beckton Dickinson, Sparks, MD., USA) as recommended by the Clinical Laboratory Standard Institute [14]. The minimum inhibitory concentrations (MICs) of cefpodoxime, cefepime, cefoxitin, aztreonam, and imipenem, and the $\beta$-lactam/ $\beta$-lactamase inhibitor combinations: cefpodoxime/clavulanate, and cefepime/clavulanate were determined by broth microdilution according to CLSI guidelines [14] using TREK microbroth dilution panels (Cleveland, Ohio, USA).

\section{B-lactamase characterization}

Crude $\beta$-lactamase extracts from the clinical isolates and strains producing reference $\beta$-lactamases were assessed for $\beta$-lactamase $\mathrm{pI}$ values, inhibitor and substrate characteristics by isoelectric focusing (IEF) [15].

\section{$\beta$-lactamase gene identification and analysis of upstream region}

PCR amplification was used to identify the presence of $b l a_{\text {CTX-M-15-like }}$ in the E. coli clinical isolate, and $b l a_{\text {CTX-M-14- }}$ like in K. pneumoniae and E. cloacae isolates using specific primers that targeted CTX-M group I and IV; respectively [16]. The presence of genes encoding TEM and SHV enzymes was analyzed by PCR [17]. The $\mathrm{MgCl}_{2}$ concentration used was $2 \mathrm{mM}$ for $b l a_{\mathrm{TEM}}$ and $b l a_{\mathrm{SHV}}$ PCR and $1.5 \mathrm{mM}$ for $b l a_{\text {CTX-M }}$ PCR. Template DNA preparation and PCR amplifications were carried out as previously described [17].

PCR amplification and sequencing of the full-length $b l a_{\text {CTX-M-14-like }}$ gene was performed, using primers that flanked the gene (CTXM14 F1 5'-GAG TGT TGC TCT GTG GAT AAC-3', designed using accession number AF252622 and annealing at positions 1857-1876; and CTX14R4 5'GTT ACA GCC CTT CGG CGA TG-3' designed using accession number AF252622 and annealing at positions 2617$2598)$. Sequence analysis of the $b l a_{\text {CTX-M-15-like gene was }}$ done using CTX3 FLF 5'-CGT CTC TTC CAG AAT AAG G3', designed using accession number AY995205 and annealing at positions 169-187; and CTX3 FLR 5'-GTT TCC CCA TTC CGT TTC CGC-3' designed using accession number AY995205 and annealing at positions 10921072).

Sequence analysis of the 524 bp upstream region of the structural gene for $b l a_{\mathrm{CTX}-\mathrm{M}-15}$ was performed on an amplified product generated using primers ISEcp1 (AGC CAA ATA CGA CAT GGC GGT G, this primer corresponds to nucleotide numbers 1179 to 1200 of the sequence with accession no. DQ658222) and CTX15 (CTT CCT AAC AAC AGC GTG AC, this primer corresponds to nucleotide numbers 261-242 of the sequence with the accession number AY995205). The 184 bases upstream of the bla $a_{\mathrm{CTX}-\mathrm{M}-14}$ were sequenced using the CTX14 upstream primer (GCA CCT GCG TAT TAT CTG C, this primer corresponds to nucleotide numbers $184-166$ of the sequence with the accession number DQ359215).

Five microliters aliquots of PCR products were analyzed by gel electrophoresis with 1\% agarose gels (BioRad, Hercules, Calif.) in TAE buffer. Gels were stained with ethidium bromide $(10 \mathrm{mg} / \mathrm{L})$ and visualized by UV transilluminator. 
The PCR products were purified with Microcon YM-50 columns (Micon bioseparations, Bedford, MS, USA). The amplicons were sequenced using automated PCR cycle sequencing with dye terminator chemistry using $\mathrm{ABI}$ PRISM 3100 Genetic Analyzer and Data collection software (version 3.7).

The nucleotide and deduced amino acid sequences were analyzed and compared using BLAST software available online at http://www.ncbi.nlm.nih.gov/BLAST.

\section{Conjugation experiments}

To determine whether the cefotaxime resistance was carried on a conjugative plasmid, conjugation experiments were performed with $K$. pneumoniae (only one isolate was tested), E. coli and E. cloacae as donors and the E. coli ( $\mathrm{Na}$ azide $^{R}$ ) as the recipient. The filter mating technique was carried out as previously described [18]. Transformants were selected on Mueller Hinton agar plates containing sodium azide $200 \mathrm{mg} / \mathrm{L}$ and cefotaxime $2 \mathrm{mg} / \mathrm{L}$ and were confirmed for $b l a_{\text {CTX-M }}$ genes using PCR as described above.

\section{Results and discussion Antimicrobial susceptibility}

Disk diffusion showed that all isolates were resistant to cefotaxime and positive for ESBL production by disk confirmatory test using cefotaxime/clavulanate and ceftazidime/clavulanate (Table 1 ). The MICs of $\beta$-lactams and $\beta$ lactam/inhibitor combinations were determined by broth microdilution technique. All clinical isolates were resistant to cefpodoxime, cefepime and resistant or intermediately resistant to aztreonam. The phenotypic ESBL microdilution confirmatory test was positive, showing a decrease by 7 doubling dilutions in the presence of clavulanic acid (Table 2). The K. pneumoniae clinical isolates were also resistant to other non- $\beta$-lactam antibiotics such as tetracycline, gentamicin and fluoroquinolones (Table $1)$.

Isolates of the Enterobacteriaceae producing CTX-M ESBLs are resistant to cefotaxime (MICs $\geq 64 \mathrm{mg} / \mathrm{L}$ ) [9] and cefepime (MICs $\geq 32 \mathrm{mg} / \mathrm{L}$ ) [19-22], but are susceptible or intermediate to ceftazidime [9]. The phenotypic characteristics of the clinical isolates in this study suggested the presence of CTX-M ESBLs. Screening using ceftazidime alone is not sufficient for organisms producing CTX-M ESBLs [16]. However, CTX-M-15 has been reported to possess some hydrolytic activity against ceftazidime [10]. The E. coli isolate producing CTX-M-15 was intermediate to ceftazidime using disk susceptibility test (Table 1).

\section{Characterization of $\beta$-lactamases}

Isoelectric focusing (IEF) of crude sonicates of the clinical isolates was done by a cefotaxime/ $\beta$-lactamase inhibitor overlay technique. Two enzymes focused at pI values of 8.0 and 9.0, were inhibited by clavulanic acid, and showed an extended spectrum of activity by hydrolyzing cefotaxime (Table 2). PCR and sequence analysis identified $b l a_{\mathrm{CTX}-\mathrm{M}-14}$ in one isolate of $K$. pneumoniae (KP 4 ) and the E. cloacae isolate, and $b l a_{\mathrm{CTX}-\mathrm{M}-15}$ in the E. coli clinical isolate (Table 2). Only one K. pneumoniae isolate was evaluated by sequence analysis because all three of the $K$. pneumoniae isolates showed the same enzymes on the IEF gel (Table 2).

All isolates produced multiple $\beta$-lactamases that were inhibited by clavulanate: $K$. pneumoniae (pI values 5.4, 6.3, 7.6, and 8.0), E. cloacae (pI values 6.3, 7.6, 8.0), and E. coli (pI values, 5.4, 6.0, 6.6 and 9.0) (Table 2). The $b l a_{\text {TEM }}$ gene was detected in all K. pneumoniae and E. coli

Table I: Susceptibility data of the clinical isolates and the transconjugant

\begin{tabular}{|c|c|c|c|c|c|c|c|c|c|}
\hline \multirow[b]{2}{*}{ Clinical Isolate } & \multicolumn{9}{|c|}{ Zone diameter of inhibition (mm) } \\
\hline & CTX & CTX/CLA & CAZ & CAZ/CLA & TET & GEN & AMK & $\mathrm{CIP}$ & SXT \\
\hline Kp4 & 6 & 18 & 18 & 25 & 6 & 8 & 20 & 6 & ND \\
\hline Kp8 & 6 & 16 & 19 & 26 & 6 & 8 & 20 & 6 & ND \\
\hline Kpl5 & 6 & 16 & 18 & 25 & 6 & 8 & 20 & 6 & ND \\
\hline ENT & 10 & 20 & 21 & 25 & 20 & 17 & 21 & 30 & ND \\
\hline EC & 9 & 22 & 15 & 26 & 19 & 19 & 20 & 32 & 6 \\
\hline TcECa & II & 31 & 20 & 32 & 25 & ND & ND & ND & 33 \\
\hline $\mathrm{EC} \mathrm{Na} \mathrm{az}{ }^{\mathrm{R}}$ & 34 & 32 & 29 & 29 & 22 & 23 & ND & 28 & 30 \\
\hline
\end{tabular}

Isolates were tested for susceptibility using disk diffusion according to CLSI guidelines [14].

CTX: cefotaxime, CTX/CLA: cefotaxime/clavulanic acid, CAZ: ceftazidime, CAZ/CLA: ceftazidime/clavulanic acid, TET: tetracycline, GEN:

gentamicin; AMK: amikacin; CIP: ciprofloxacin, SXT: sulfamethoxazole/trimethoprim,

TcEC: transconjugant carrying plasmid encoding bla CTX-M-15.

$E C N a z^{R}$ : recipient strain resistant to sodium azide

a Transconjugant was tested by PCR experiment for bla ${ }_{\text {CTX-M-15-like }}$ using specific primers [16].

ND: Not determined 
Table 2: MIC data of selected $\beta$-lactams and characteristics of $\beta$-lactamases produced by clinical isolates of Enterobacteriaceae from Egypt

\begin{tabular}{|c|c|c|c|c|c|c|c|c|c|c|c|c|}
\hline \multirow[t]{3}{*}{ Clinical Isolate } & \multicolumn{4}{|c|}{ Enzyme characteristics $^{a}$} & \multirow[t]{3}{*}{ Gene (s) detected by PCR ${ }^{b}$} & \multicolumn{7}{|c|}{ MIC (mg/L) of: } \\
\hline & \multirow[t]{2}{*}{$\mathrm{pl}$} & \multirow[t]{2}{*}{ CTX hydrolysis } & \multicolumn{2}{|c|}{ Inhibited by } & & \multirow[t]{2}{*}{ CPD } & \multirow[t]{2}{*}{ CPD/CLA } & \multirow[t]{2}{*}{ FEP } & \multirow[t]{2}{*}{ FEP/CLA } & \multirow[t]{2}{*}{ FOX } & \multirow[t]{2}{*}{ ATM } & \multirow[t]{2}{*}{ IPM } \\
\hline & & & Clox & CLA & & & & & & & & \\
\hline Kp4 & 5.4 & No & No & Yes & $b l a_{\mathrm{TEM}}$ & $>128$ & 1 & $>128$ & 0.06 & 8 & 128 & 0.12 \\
\hline Kp8 & 6.3 & No & No & Yes & $-c$ & $>128$ & 2 & $>128$ & 0.06 & 8 & 128 & 0.12 \\
\hline \multirow[t]{2}{*}{ KpI5 } & 7.6 & No & No & Yes & $b l a_{\mathrm{SHV}}$ & $>128$ & 2 & $>128$ & $<0.03$ & 8 & 128 & 0.12 \\
\hline & 8.0 & Yes & No & Yes & bla CTX-M-14 & & & & & & & \\
\hline \multirow[t]{4}{*}{ ENT } & 6.3 & No & No & Yes & $-c$ & $>128$ & 2 & $>128$ & $<0.03$ & $>16$ & 32 & 0.25 \\
\hline & 7.6 & No & No & Yes & $-e$ & & & & & & & \\
\hline & 8.0 & Yes & No & Yes & $b l a_{\text {CTX-M- } 14}$ & & & & & & & \\
\hline & 8.9 & No & Yes & No & $-d$ & & & & & & & \\
\hline \multirow[t]{4}{*}{ EC } & 5.4 & No & No & Yes & $b l a_{\mathrm{TEM}}$ & $>128$ & 0.25 & $>128$ & $<0.03$ & $<4$ & 16 & 0.12 \\
\hline & 6.0 & No & No & Yes & $-c$ & & & & & & & \\
\hline & 6.6 & No & No & Yes & $-c$ & & & & & & & \\
\hline & 9.0 & Yes & No & Yes & $b l a_{\mathrm{CTX}-\mathrm{M}-15}$ & & & & & & & \\
\hline
\end{tabular}

CPD: cefpodoxime, CPD/CLA: cefpodoxime/clavulanic acid, FEP: cefepime, FEP/CLA: cefepime/clavulanic acid, FOX: cefoxitin, ATM: aztreonam, IPM: imipenem.

${ }^{a}$ Enzyme Characteristics: pl: isoelectric point of crude $\beta$-lactamase extract preparations; CTX $(0.75 \mathrm{mg} / \mathrm{L})$ was used in the substrate-based IEF overlay technique, inhibitors used in the IEF overlay were clavulanic acid $(\mathrm{I} \mathrm{mM})$ and cloxacillin $(\mathrm{I} \mathrm{mM})$.

${ }^{b}$ bla $_{\mathrm{TEM}}$, bla $a_{\mathrm{SHV}}$ and bla $a_{\mathrm{CTX}-\mathrm{M}}$ genes were detected by PCR experiments using specific primers. Only the bla ${ }_{\mathrm{CTX}-\mathrm{M}}$ genes were sequenced using primers that flanked the full-length genes (See Methods section).

c PCR was not done to detect the gene that corresponds to the $\beta$-lactamase band on IEF gel.

d $\beta$-lactamase band focusing at pl value of 8.9 , which is inhibited by cloxacillin, corresponds to the chromosomal ampC gene of $E$. cloacae (PCR data not shown).

e PCR was negative

isolates, and corresponded to the $\beta$-lactamase band that focused at pI value of 5.4 when evaluated by IEF (Table 2). The $b l a_{\mathrm{SHV}}$ gene was present in the K. pneumoniae isolates ( $\beta$-lactamase band focusing at $\mathrm{pI}$ value, 7.6-Table 2 ). The $\beta$-lactamase band that focused at pI value of 7.6 in the $E$. cloacae isolate was most likely not an SHV-enzyme since SHV-specific PCR was negative. Further sequencing experiments for the $b l a_{\mathrm{TEM}}$ and $b l a_{\mathrm{SHV}}$ genes were not done.

Analysis of the upstream sequence of $b l a_{\mathrm{CTX}-\mathrm{M}-14}$ and of $b l a_{\text {CTX-M-15 }}$ revealed the presence of the right terminal inverted repeat of the insertion sequence ISEcp 1 and the putative promoter region $(-10$ and -35$)$ associated with this element [23].

The results of the conjugation experiment showed that $b l a_{\mathrm{CTX}-\mathrm{M}-15}$ was carried on a conjugative plasmid (Table 1 ). The movement of $b l a_{\mathrm{CTX}-\mathrm{M}-15}$ was verified in the transconjugant using CTX-M-group 1-specific PCR [16]. The bla $a_{\text {CTX- }}$ $\mathrm{M}-14$ gene was not mobilized by conjugation.

A surveillance report on antibiotic resistance in the Southeastern Mediterranean region screened only E. coli isolates from different medical centers in Egypt [2]. Other important nosocomial isolates such as $K$. pneumoniae and $E$. cloacae were not evaluated in that study [2]. A recent outbreak was reported in a neonatal intensive care unit in
Cairo, Egypt, in which $80 \%$ of the isolates were K. pneumoniae, of which $58 \%$ were ESBL producers [24]. Therefore, it is important not to limit extended-spectrum cephalosporin susceptibility screening in Egypt to E. coli but to include K. pneumoniae as well as other Enterobacteriaceae such as E. cloacae.

It is important for clinical microbiologists in Egyptian hospitals to screen for CTX-M ESBL producers. In addition, clinical microbiologists and physicians need to be aware that these enzymes are present in many different types of Enterobacteriaceae. This information is essential for determining the most appropriate empirical antibiotic therapy.

\section{Conclusion}

This study is the first documentation of CTX-M-14 ESBLs in K. pneumoniae and E. cloacae isolates in Egypt as well as the Middle East region and North Africa.

\section{Competing interests}

The authors declare that they have no competing interests.

\section{Authors' contributions}

NK participated in the design of the study. NK carried out the susceptibility testing, molecular genetic studies, and sequence alignment; and participated in drafting the man- 
uscript. NDH participated in the design of the study and drafting the manuscript, and coordination. Sequencing primers were designed by NDH. Work was carried out at the laboratory of Dr. Hanson, Department of Microbiology and Immunology, Creighton University, Omaha, Nebraska. All authors have analyzed and interpreted the data, and have read and approved the final manuscript.

\section{Acknowledgements}

We wish to thank Ellen Smith-Moland at the department of Microbiology and Immunology, Creighton University, Omaha, Nebraska for advice on the susceptibility testing, her valuable help in performing the isoelectric focusing experiments, and for her helpful discussions during the progress of the work. The Committee for Protection of Human Subjects at the medical institute has approved the scientific protocol and specimens were obtained from consenting patients. We thank George Jacoby for providing the $\mathrm{Na}$ azide $^{R} E$. coli strain. Sequence data analysis was supported by a grant from the Binational Fulbright Commission (Egypt/USA).

\section{References}

I. El Kholy A, Baseem H, Hall GS, Procop GW, Longworth DL: Antimicrobial resistance in Cairo, Egypt 1999-2000: a survey of five hospitals. J Antimicrob Chemother 2003, 5 I (3):625-630.

2. Borg MA, Scicluna E, de Kraker M, Sande-Bruinsma N van de, Tiemersma E, Gur D, Ben Redjeb S, Rasslan O, Elnassar Z, Benbachir M, et al: Antibiotic resistance in the southeastern Mediterranean - preliminary results from the ARMed project. Euro Surveill 2006, I I(7): 164-167.

3. Bouchillon SK, Johnson BM, Hoban DJ, Johnson JL, Dowzicky MJ, Wu $\mathrm{DH}$, Visalli MA, Bradford PA: Determining incidence of extended spectrum beta-lactamase producing Enterobacteriaceae, vancomycin-resistant Enterococcus faecium and methicillin-resistant Staphylococcus aureus in 38 centres from 17 countries: the PEARLS study 200 I-2002. Int J Antimicrob Agents 2004, 24(2): I 19-124.

4. Paterson DL, Bonomo RA: Extended-spectrum beta-lactamases: a clinical update. Clin Microbiol Rev 2005, 18(4):657-686.

5. Mohamed Al-Agamy MH, El-Din Ashour MS, Wiegand I: First description of CTX-M beta-lactamase-producing clinical Escherichia coli isolates from Egypt. Int J Antimicrob Agents 2006, 27(6):545-548.

6. Moubareck C, Daoud Z, Hakime NI, Hamze M, Mangeney N, Matta H, Mokhbat JE, Rohban R, Sarkis DK, Doucet-Populaire F: Countrywide spread of community- and hospital-acquired extendedspectrum beta-lactamase (CTX-M-I5)-producing Enterobacteriaceae in Lebanon. J Clin Microbiol 2005, 43(7):3309-33। 3.

7. Touati A, Benallaoua S, Forte D, Madoux J, Brasme L, de Champs C: First report of CTX-M-I5 and CTX-M-3 beta-lactamases among clinical isolates of Enterobacteriaceae in Bejaia, Algeria. Int J Antimicrob Agents 2006, 27(5):397-402.

8. Jouini A LV, Ben Slama K, Sáenz Y, Klibi , Hammami S, Boudabous A, Torres Carmen: Characterization of CTX-M and SHV extended-spectrum beta-lactamases and associated resistance genes in Escherichia coli strains of food samples in Tunisia. J Antimicrob Chemother 2007, 60: I I37-II4I.

9. Bonnet R: Growing group of extended-spectrum beta-lactamases: the CTX-M enzymes. Antimicrob Agents Chemother 2004, 48(I): $1-14$.

10. Poirel L, Gniadkowski M, Nordmann P: Biochemical analysis of the ceftazidime-hydrolysing extended-spectrum beta-lactamase CTX-M-I5 and of its structurally related beta-lactamase CTX-M-3. J Antimicrob Chemother 2002, 50(6): I03।-1034.

II. Poirel L, Naas T, Le Thomas I, Karim A, Bingen E, Nordmann P: CTXM-type extended-spectrum beta-lactamase that hydrolyzes ceftazidime through a single amino acid substitution in the omega loop. Antimicrob Agents Chemother 200 I 45(I 2):3355-336I.

12. Poirel L, Kampfer P, Nordmann P: Chromosome-encoded Ambler class A beta-lactamase of Kluyvera georgiana, a probable progenitor of a subgroup of CTX-M extended- spectrum beta-lactamases. Antimicrob Agents Chemother 2002, 46(I2):4038-4040.

13. Humeniuk C, Arlet G, Gautier V, Grimont P, Labia R, Philippon A: Beta-lactamases of Kluyvera ascorbata, probable progenitors of some plasmid-encoded CTX-M types. Antimicrob Agents Chemother 2002, 46(9):3045-3049.

14. CLSI: Performance Standards for Antimicrobial Disk Susceptibility Tests-Ninth Edition: Approved Standard M-2-A9. Clinical Laboratory Standards Institute (CLSI), Wayne, PA, USA; 2006.

15. Sanders CC, Sanders WE Jr, Moland ES: Characterization of betalactamases in situ on polyacrylamide gels. Antimicrob Agents Chemother 1986, 30(6): $951-952$.

16. Pitout JD, Hossain A, Hanson ND: Phenotypic and molecular detection of CTX-M-beta-lactamases produced by Escherichia coli and Klebsiella spp. J Clin Microbiol 2004, 42( I 2):57| 5-572I.

17. Hanson ND, Thomson KS, Moland ES, Sanders CC, Berthold G, Penn RG: Molecular characterization of a multiply resistant Klebsiella pneumoniae encoding ESBLs and a plasmid-mediated AmpC. J Antimicrob Chemother 1999, 44(3):377-380.

18. Pitout JD, Thomson KS, Hanson ND, Ehrhardt AF, Coudron P, Sanders CC: Plasmid-mediated resistance to expanded-spectrum cephalosporins among Enterobacter aerogenes strains. Antimicrob Agents Chemother 1998, 42(3):596-600.

19. Yu WL, Pfaller MA, Winokur PL, Jones RN: Cefepime MIC as a predictor of the extended-spectrum beta-lactamase type in Klebsiella pneumoniae, Taiwan. Emerg Infect Dis 2002, 8(5):522-524

20. Moland ES, Black JA, Hossain A, Hanson ND, Thomson KS, Pottumarthy S: Discovery of CTX-M-like extended-spectrum betalactamases in Escherichia coli isolates from five US States. Antimicrob Agents Chemother 2003, 47(7):2382-2383.

21. Bouallegue-Godet O, Ben Salem Y, Fabre L, Demartin M, Grimont PAD, Mzoughi R, Weill FX: Nosocomial Outbreak Caused by Salmonella enterica Serotype Livingstone Producing CTXM-27 Extended-Spectrum beta-Lactamase in a Neonatal Unit in Sousse, Tunisia. J Clin Microbiol 2005, 43(3): $1037-1044$.

22. Schlesinger J, Navon-Venezia S, Chmelnitsky I, Hammer-Munz $O$, Leavitt A, Gold HS, Schwaber MJ, Carmeli Y: Extended-spectrum beta-lactamases among Enterobacter isolates obtained in Tel Aviv, Israel. Antimicrob Agents Chemother 2005, 49(3): $1150-1156$

23. Poirel L, Decousser JW, Nordmann P: Insertion sequence ISEcpIB is involved in expression and mobilization of a bla(CTX-M) beta-lactamase gene. Antimicrob Agents Chemother 2003, 47(9):2938-2945.

24. Moore KL, Kainer MA, Badrawi N, Afifi S, Wasfy M, Bashir M, Jarvis WR, Graham TW, el-Kholy A, Gipson R, et al.: Neonatal sepsis in Egypt associated with bacterial contamination of glucosecontaining intravenous fluids. Pediatr Infect Dis J 2005, 24(7):590-594.

\section{Pre-publication history}

The pre-publication history for this paper can be accessed here:

http://www.biomedcentral.com/1471-2334/9/84/prepub 